\title{
MiR-181a Targets PHLPP2 to Augment AKT Signaling and Regulate Proliferation and Apoptosis in Human Keloid Fibroblasts
}

\author{
Zhen Rang ${ }^{\mathrm{a}}$ Zong-yang Wang ${ }^{\mathrm{b}}$ Qiu-yu Pang ${ }^{\mathrm{a}}$ You-wei Wang ${ }^{\mathrm{a}}$ Ge Yang ${ }^{\mathrm{a}}$ Fan Cui \\ Institute of Dermatology, Sichuan Academy of Medical Sciences and Sichuan Provincial People's \\ Hospital, School of Medicine, University of Electronic Science and Technology of China, Chengdu, \\ bDepartment of Dermatology, Southwest Medical University Luzhou, Luzhou, China
}

\section{Key Words}

miR-181a $・$ PHLPP2 $•$ AKT $・$ Keloid fibroblasts $・$ Proliferation $•$ Apoptosis

\begin{abstract}
Background/Aims: Keloids are fibrous overgrowths induced by cutaneous injury. MicroRNAs (miRNAs) have recently emerged as post-transcriptional gene repressors and participants in a diverse array of pathophysiological processes leading to skin disease. The purpose of the current study was to explore the precise functions of miR-181a in human keloid development and the underlying mechanisms. Methods: A miRNA microarray analysis was performed to compare expression profiles between keloid and normal skin tissues. Quantitative real-time PCR was conducted to estimate miR-181a expression. Cell proliferation was determined using the cell counting kit-8 (CCK-8) and 5-ethynyl-2-deoxyuridine (EdU) assays, and cell cycle and apoptosis were detected with flow cytometry. Direct targets of miR-181a were identified using the luciferase reporter assay. Results: miR-181a was significantly upregulated in human keloid tissues and fibroblasts, compared with their control counterparts. Overexpression of miR-181a enhanced keloid fibroblast DNA synthesis and proliferation and inhibited apoptosis, whereas miR-181a suppression triggered the opposite effects. Moreover, miR-181a suppressed the expression of $\mathrm{PH}$ domain leucine-rich repeat protein phosphatase 2 (PHLPP2) through direct interactions with its 3'UTR region and subsequently enhanced AKT activation. Overexpression of PHLPP2 without its 3'UTR attenuated the effects of miR-181a on cell proliferation and apoptosis in keloid fibroblast cells. Furthermore, miR-181a mimics increased normal skin fibroblast proliferation. Conclusions: Our results highlight a novel pathway mediated by miR$181 a$, which may be effectively used as a therapeutic target for treatment of keloids.
\end{abstract}

\section{Introduction}

Keloids are benign skin tumors characterized by histological accumulation of fibroblasts and excessive deposition of extracellular matrix (ECM) components that arise 


\section{Cellular Physiology Cell Physiol Biochem 2016;40:796-806 \begin{tabular}{l|l|l} 
and BOI: 10.1159/000453139 & $\begin{array}{l}\text { C) 2016 The Author(s). Published by S. Karger AG, Basel } \\
\text { www.karger.com/cpb }\end{array}$
\end{tabular} \\ Rang et al.: MiR-181a Regulates Keloid Fibroblast Proliferation and Apoptosis}

as a consequence of abnormal wound healing processes after skin injury [1,2]. Although keloid is a benign dermal tumor, its management remains an excessively challenging clinical problem. Keloids do not regress with time, and surgical excision alone is associated with high rates of recurrence $[1,3]$. Keloid disease is a recurrent fibroproliferative cutaneous tumor of unknown pathogenesis for which clinical management remains unsatisfactory. Elucidation of the etiopathogenesis and molecular mechanisms of keloid formation may facilitate the development of effective molecule-targeted therapies.

MicroRNAs (miRNAs) are noncoding, single-stranded small RNAs 21-25 nucleotides in length that mediate post-transcriptional repression to perform important cellular functions. MiRNAs partly suppress the expression of target genes through binding to their $3^{\prime}$ untranslated regions (UTR) and consequently influence a variety of physiological and pathological processes, including proliferation, metabolism, apoptosis, and invasion [4-6]. Emerging evidence has shown that miRNAs contribute to hypertrophic scarring or keloid formation [7-9]. Earlier, Zhang et al. [7] reported that miR-29a directly regulates the translation of various ECM mRNAs, such as the collagen superfamily, in fibrogenesis of keloids, suggesting utility as a novel marker for keloid. Downregulation of miR-125b in psoriatic skin has been shown to contribute to keratinocyte hyperproliferation [8]. Another miRNA microarray study focusing on differentially expressed miRNAs between keloid and normal skin tissue revealed that several miRNAs participate in the molecular network of keloid pathogenesis through regulating expression of their target genes [9], of which only a fraction have been functionally characterized. In the current investigation, miRNA microarray analysis (employed to identify differentially expressed miRNAs between keloid and normal skin tissues) revealed a significant increase in miR-181a in keloid tissues. However, the functional roles of miR-181a and the relevant signaling pathways in the pathogenesis of human keloid fibroblasts remain to be validated.

Here, we confirmed upregulation of miR-181a in keloid tissue and keloid fibroblasts, compared with normal skin tissue and fibroblasts, respectively. Notably, miR-181a suppressed PHLPP2 expression, leading to activation of the AKT pathway, and consequently, acceleration of proliferation and suppression of apoptosis in human keloid fibroblasts. miR181a overexpression additionally enhanced the ability of normal fibroblasts to synthesize DNA. Our collective findings highlight potential biomarkers and targets that may be applied in diagnostic and therapeutic approaches for keloids.

\section{Materials and Methods}

\section{Patient Samples}

Twenty paired keloid tissue and normal skin tissue samples were obtained from patients with keloids on the main chest, ear lobe, shoulder and thigh, and diagnosed pathologically at the Department of Dermatology of Sichuan Provincial People's Hospital. All experiments were approved by the Ethics Committee of Sichuan Academy of Medical Sciences \& Sichuan Provincial People's Hospital (China) and written informed consent was obtained for each patient. Collection of skin samples was carried out in accordance with the approved guidelines. The skin samples collected were used for histopathological examination, preparation of total RNA and total protein lysates, and isolation and culture of fibroblasts.

\section{Cell culture}

Cultures of keloid and normal skin fibroblasts isolated from keloid tissue and normal skin samples were established as described previously $[1,10]$. Keloid samples were treated with PBS containing ampicillin and streptomycin sulfate. Epidermis and subcutaneous fat were washed away from keloid samples using PBS. Next, keloid samples $\left(\sim 1 \mathrm{~mm}^{3}\right)$ were incubated in culture flasks containing Dulbecco's Modified Eagle Medium (DMEM), $200 \mathrm{U} / \mathrm{ml}$ collagenase and 300U/ml hyaluronidase. Cells were cultured in DMEM supplemented with $10 \%$ fetal bovine serum in an atmosphere containing $5 \% \mathrm{CO}_{2}$. 


\section{Cellular Physiology Cell Physiol Biochem 2016;40:796-806 \begin{tabular}{l|l|l|} 
and BOI: 10.1159/000453139 & $\begin{array}{l}\text { C) 2016 The Author(s). Published by S. Karger AG, Basel } \\
\text { www.karger.com/cpb }\end{array}$
\end{tabular} \\ Rang et al.: MiR-181a Regulates Keloid Fibroblast Proliferation and Apoptosis}

Expression profiles of miRNAs in keloid tissue

Total RNAs were extracted from three paired samples of keloid and normal skin tissue using the miRcute miRNA Isolation Kit (Qiagen, Germany). In total, 500 ng RNA was subjected to the Agilent miRNA microarray analysis service (LC Sciences, Houston, TX, USA). Data analysis was performed with GenePix Pro software (LC Sciences) [11]. Statistical significance was examined using Student's $t$-test, and values of $P<0.05$ considered significant.

\section{Western blot analysis}

Western blotting was performed as described previously [12]. Total proteins were extracted from keloid tissues and fibroblasts using RIPA lysis buffer, separated using 10\% sodium dodecyl sulfatepolyacrylamide gel electrophoresis, and transferred onto polyvinylidene difluoride membrane (Roche Life Sciences, Switzerland). Membranes were blocked with 5\% skimmed milk and incubated with the appropriate antibodies. The anti-PHLPP2 antibody was obtained from Abcam, and phosphorylated Akt and AKT antibodies acquired from Cell Signaling Technology (Danvers, MA, USA). Primary antibodies were applied to the membrane, followed by horseradish-peroxidase-conjugated secondary antibodies. The antigen-antibody complex on the membrane was detected with enhanced chemiluminescence reagent (Thermo Scientific, Waltham, MA, USA).

\section{Quantitative RT-PCR}

Total RNA was obtained from cell lines using TRIzol reagent (Invitrogen, Carlsbad, CA, USA). Complementary DNA synthesis was performed using the PrimeScript reverse transcriptase reagent kit (Takara, Dalian, China) according to the manufacturer's protocol. qRT-PCR was conducted to detect the expression levels of miR-181a and PHLPP2 using SYBR ${ }^{\circledR}$ Premix ExTaq (Takara). U6 or GAPDH served as the internal control for miRNA and mRNA.

\section{Cell transfection}

All synthetic miRNAs, including miR-181a mimic, miR-181a inhibitor and the respective controls, were purchased from Shanghai Genepharma (Shanghai, China). Human keloid fibroblasts were transfected with miR-181a mimic or miR-181a inhibitor using Lipofectamine 2000 (Invitrogen) according to the manufacturer's instructions, and cells harvested after 24,48 , and $72 \mathrm{~h}$ to examine transfection efficiency. All procedures were performed in triplicate.

Reporter vector construction and luciferase reporter assay

PHLPP2 wild-type (Wt) and mutant (Mut) 3'UTR were created and cloned into psiCHECK-2 luciferase reporter plasmid (Promega). For the luciferase assay, cells were plated intosix-well plates the day before transfection and cotransfected with the PHLPP2 Wt or Mut 3'UTR reporter vector and miR-181amimic or scramble control using Lipofectamine 2000 (Invitrogen). Luciferase activities were determined with the Dual-Luciferase Reporter System (Promega). All assays were performed in triplicate.

\section{Cell proliferation assay}

Human keloid fibroblast cell proliferation was measured using the CCK-8 (Beyotime Institute of Biotechnology, Jiangsu, China) and 5-ethynyl-2'-deoxyuridine (EdU) incorporation assay (Life Technologies Corporation, Carlsbad, CA, USA) kits, in keeping with the manufacturer's instructions. For the CCK-8 assay, cells were seeded into a 96-well plate andCCK-8 solution added. For the EdU incorporation assay, $5 \times 10^{3}$ cells/well were seeded into 96-well plates, transfected for $24 \mathrm{~h}$, and incubated with EdU for $2 \mathrm{~h}$ [13]. The EdU incorporation rate was expressed as the ratio of EdU-positive to total Hoechst 33342-positive cells (blue cells).

\section{Cell cycle and apoptosis assays}

Cell cycle analysis was performed with a cell cycle detection kit (KeyGen Biotech, Nanjing, China) and flow cytometry. Cells were transfected with a miR-181a mimic or inhibitor for $24 \mathrm{~h}$, and subsequently collected, washed, resuspended and incubated with annexin V-fluorescein isothiocyanate/propidium iodide in the dark for $15 \mathrm{~min}$, followed byflow cytometry analysis using FloMax software (Partec, Munich,Germany). The cell population fraction was assessed using quadrant statistics [14]. 


\section{Cellular Physiology Cell Physiol Biochem 2016;40:796-806

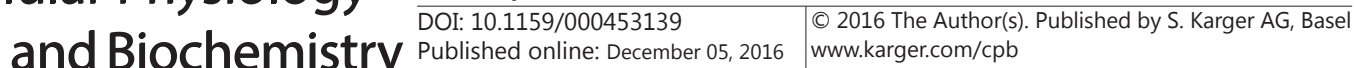 \\ Rang et al.: MiR-181a Regulates Keloid Fibroblast Proliferation and Apoptosis}

\section{Statistical analysis}

Data are expressed as means \pm standard deviation. Statistical analyses were performed using GraphPad Prism 5.0. Statistical significance was determined using Student's $t$-test when only two groups were compared or one-way analysis of variance (ANOVA) test when more than two groups were compared. $P$ values of less than 0.05 were considered significant.

\section{Results}

miR-181a is upregulated in human keloid tissues and fibroblasts

Differentially expressed miRNAs between three keloid and normal skin tissue sets were identified via miRNA microarray (Table 1). In particular, we focused on miR-181a, which displayed significantly increased expression in keloid tissues (Fig. 1A). To further validate the miRNA microarray results, we evaluated miR-181a expression in 20 pairs of keloid and matched normal skin tissues, and additionally in keloid and normal fibroblasts, using qRTPCR. As anticipated, miR-181a expression was markedly upregulated in both keloid tissues and fibroblasts, compared to the normal skin and fibroblast counterparts, respectively (Fig. $1 \mathrm{~B}, \mathrm{C})$.

\section{miR-181a enhances human keloid fibroblast DNA synthesis and proliferation}

To explore the biological impact of miR-181a on human keloid fibroblasts, we performed gain- and loss-of-function experiments by transfecting keloid fibroblasts with a miR181a mimic and miR-181a inhibitor, respectively. The transfection efficiency of miR-181a was determined after 24,48 , and $72 \mathrm{~h}$ using qRT-PCR. miR-181a expression was remarkably enhanced in keloid fibroblasts transfected with the miR-181a mimic, compared with the scramble control, while expression was significantly decreased in cells transfected with the miR-181a inhibitor, relative to the negative control (Fig. 2A). Efficiency of transfection was not significantly different between the different time-points. The CCK-8 assay was employed to define the effects of miR-181a on cell proliferation. Our data revealed a significant increase in viability of miR-181a mimic-treated keloid fibroblasts than the scramble control group and suppression of viability of miR-181a inhibitor-treated cells, compared with the negative control group (Fig. 2B). We additionally performed the EdU incorporation assay to confirm the function of miR-181a in cell proliferation. The number of EdU-positive keloid fibroblasts in the miR-181a mimic group was enhanced relative to that in cells from the scramble control group, and the EdU-positive keloid fibroblast number in the miR-181a inhibitor group was reduced, compared with the negative control group (Fig. 2C). Consistently, expression of proliferation-related protein PCNA was increased in keloid fibroblasts transfected with the miR-181a mimic, and conversely, suppressed in cells transfected with the miR-181a inhibitor (Fig. 2D). Furthermore, overexpression of miR-181a also promoted the cell cycle, and a decrease in the G0/ G1 fraction was observed in human keloid fibroblasts transfected with the miR181a mimic. Treatment

Table 1. Differentially expressed miRNAs with $\mathrm{P}<0.05$

\begin{tabular}{lllll}
\hline No. & miRNA-ID & Regulation & Fold Change & P-value (t-test) \\
\hline 1 & hsa-miR-181a-5p & up & 6.044 & 0.023 \\
2 & hsa-miR-181b-5p & up & 3.100 & 0.030 \\
3 & hsa-miR-21-3p & up & 24.415 & 0.007 \\
4 & hsa-miR-21-5p & up & 9.311 & 0.029 \\
5 & hsa-miR-1260b & up & 3.389 & 0.009 \\
6 & hsa-miR-127-3p & up & 5.276 & 0.025 \\
7 & hsa-miR-132-3p & up & 3.208 & 0.025 \\
8 & hsa-miR-146b-5p & up & 2.927 & 0.027 \\
9 & hsa-miR-152 & up & 4.268 & 0.005 \\
10 & hsa-miR-5684 & up & 4.387 & 0.026 \\
11 & hsa-miR-1207-5p & down & 3.307 & 0.031 \\
12 & hsa-miR-1587 & down & 4.133 & 0.015 \\
13 & hsa-miR-188-5p & down & 2.170 & 0.017 \\
14 & hsa-miR-3188 & down & 2.044 & 0.022 \\
15 & hsa-miR-1234-5p & down & 2.635 & 0.039 \\
16 & hsa-miR-2392 & down & 2.170 & 0.014 \\
17 & hsa-miR-3194-5p & down & 2.736 & 0.039 \\
18 & hsa-miR-3679-5p & down & 2.085 & 0.018 \\
19 & hsa-miR-3937 & down & 2.086 & 0.044 \\
20 & hsa-miR-3960 & down & 2.595 & 0.032 \\
\hline
\end{tabular}




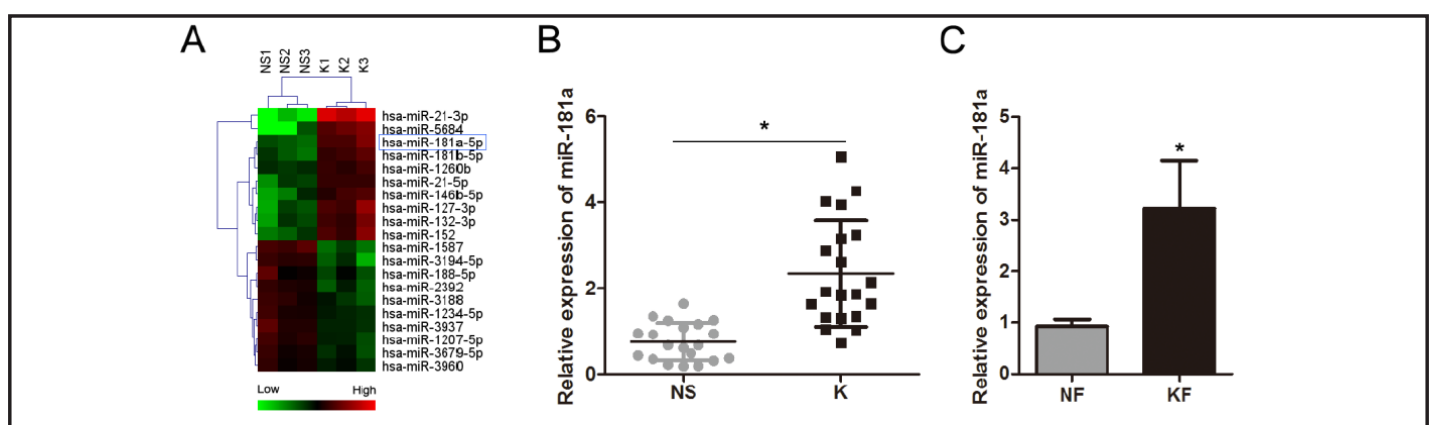

Fig. 1. miR-181a expression is increased in human keloid tissues and fibroblasts. (A) Heatmap of 20 miRNAs displaying significant upregulation or downregulation in human keloid tissues, compared with matched normal skin tissues. (B) qRT-PCR analysis ofmiR-181a expression in 20 pairs of normal skin(NS) and keloid tissues(K). (C) qRT-PCR analysis of miR-181a expression innormal fibroblasts (NF) and keloid fibroblasts $(\mathrm{KF}),{ }^{*} P<0.05$.

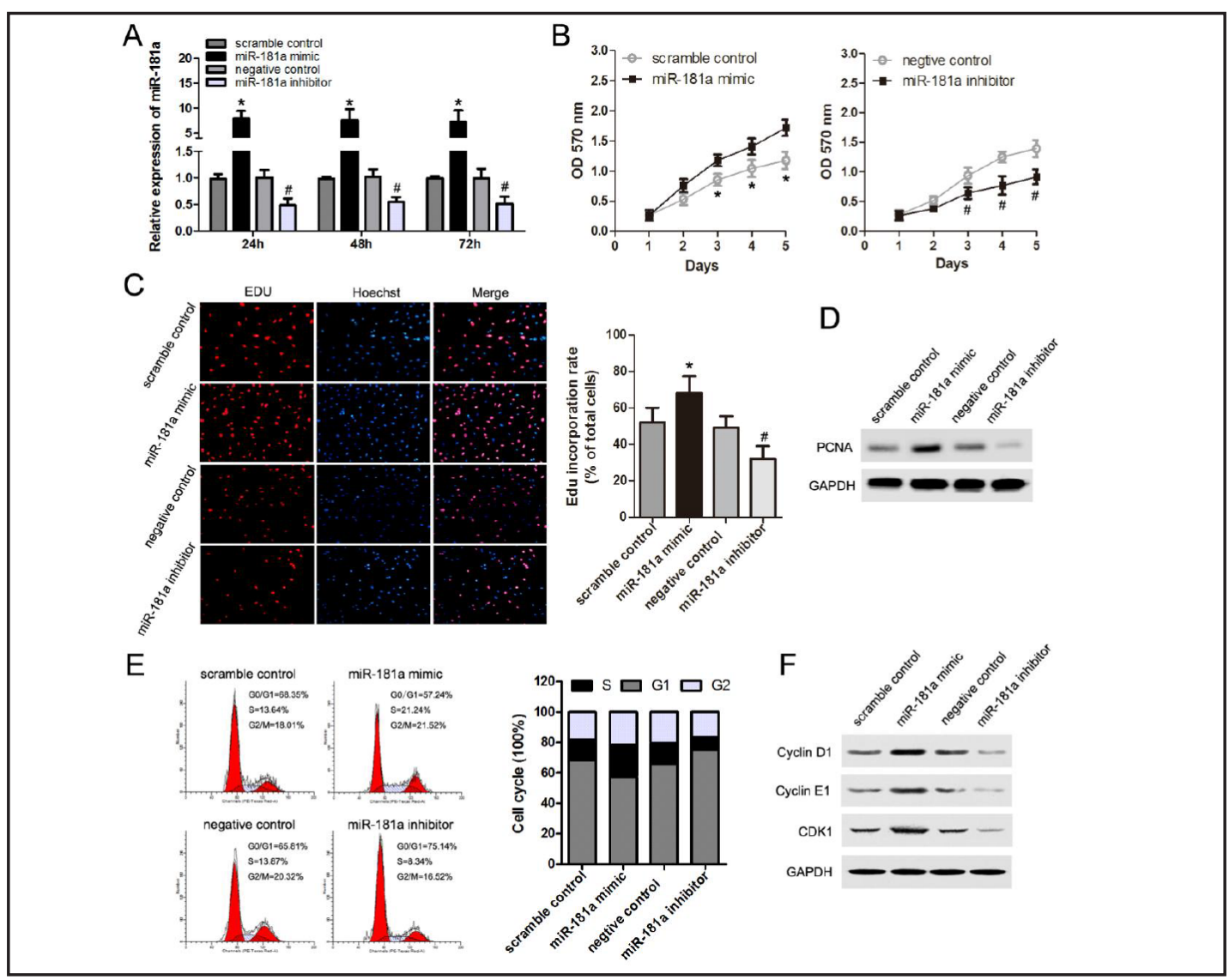

Fig. 2. miR-181a enhances human keloid fibroblast DNA synthesis and proliferation. (A) qRT-PCR determination of the relative miR-181a expression levels in $\mathrm{KF}$ at 24, 48 and $72 \mathrm{~h}$ after transfection with miR-181a mimic, miR-181a inhibitor or control miRNA. (B) Cell proliferation abilities of keloid fibroblasts after transfection with miR-181a mimic or inhibitor determined using the CCK-8 assay. (C) EdU incorporation assay to evaluate the effects of miR-181a mimic and inhibitor on cell proliferation of keloid fibroblasts. The EdU incorporation rate was expressed as the ratio of EdU-positive to total Hoechst 33342-positive cells. (D) Western blot analysis of expression of cell proliferation-related molecules $24 \mathrm{~h}$ after transfection with the miR181a mimic or miR-181a inhibitor and the respective controls. (E) Flow cytometry evaluation of the cell cycle after transfection of keloid fibroblasts with miR-181a mimic or inhibitor. (F) Western blot analysis of expression of cell cycle-related molecules $24 \mathrm{~h}$ after transfection with the miR-181a mimic or miR-181a inhibitor and the respective controls. ${ }^{*} P<0.05$ vs. scramble control group; ${ }^{\#} P<0.05$ vs. negative control group. 


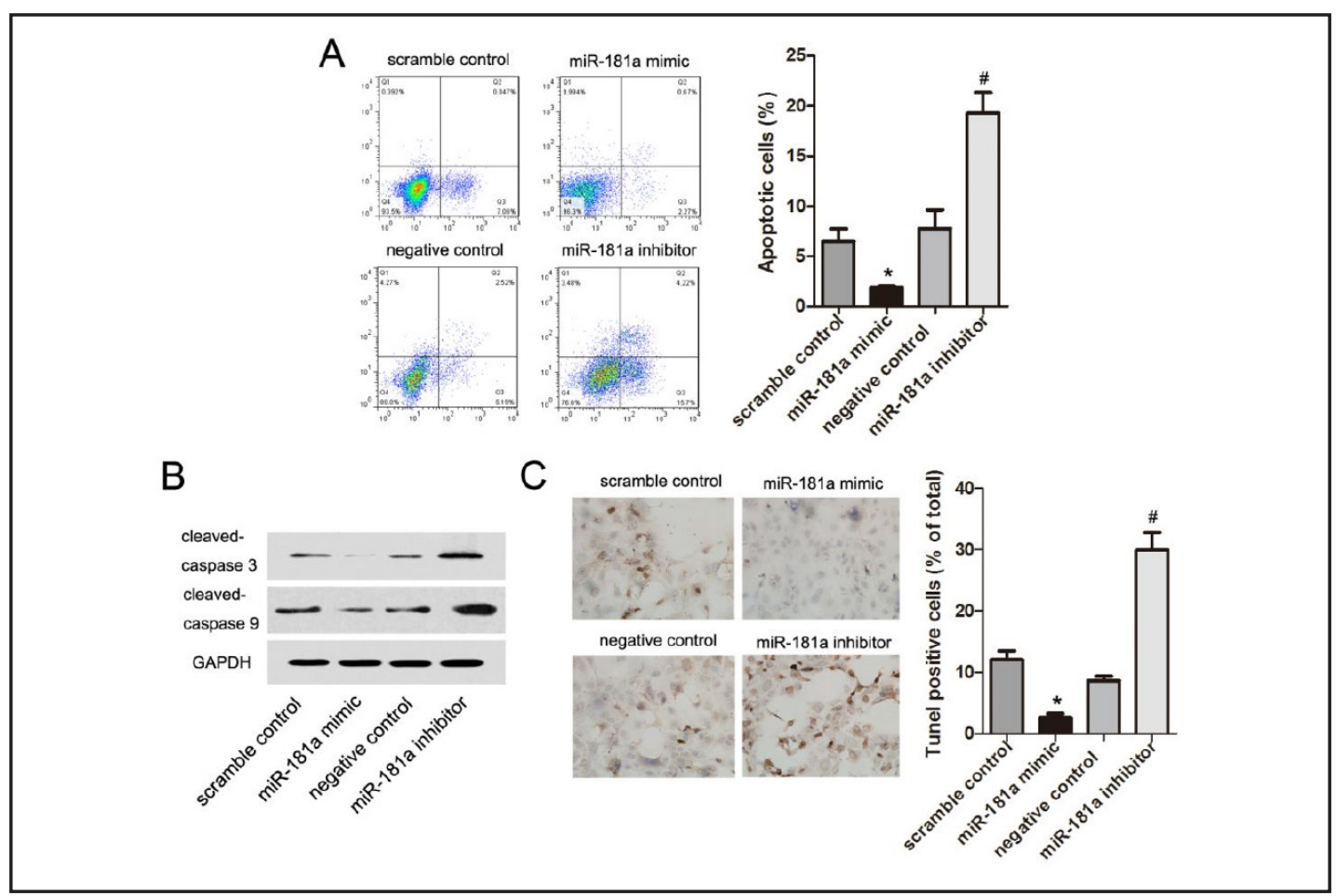

Fig. 3. miR-181a inhibits human keloid fibroblast apoptosis. (A) Flow cytometry measurement of cell apoptosis and quantification after transfection of keloid fibroblasts with miR-181a mimic or inhibitor and their respective controls. (B) Western blot analysis of cleaved caspase- 3 and caspase- 9 expression in keloid fibroblasts transfected with miR-181a mimic or inhibitor and their respective controls. (C) TUNEL staining to detect apoptosis in keloid fibroblasts transfected with miR-181a inhibitor or mimic. ${ }^{*} P<0.05$ vs. scramble control group; ${ }^{\#} P<0.05$ vs. negative control group.

with the miR-181a mimic led to a concomitant increase in the cell population in the S phase. Opposite results were obtained with the miR-181a inhibitor (Fig. 2E). Enhanced expression levels of cell cycle-related proteins, including cyclin D1, cyclin E1 and CDK1 in keloid fibroblasts transfected with the miR-181a mimic and reduced in keloid fibroblasts transfected with the miR-181a inhibitor further confirmed that miR-181a promoted the cell cycle of keloid fibroblasts (Fig. 2F). Our data clearly indicate that aberrant expression of miR-181a contributes to proliferation of keloid fibroblasts.

\section{miR-181a inhibits human keloid fibroblast apoptosis}

Next, we analyzed the effects of miR-181a on apoptosis using flow cytometry and western blot analyses. As shown in Fig. $3 \mathrm{~A}$ and B, the miR-181a mimic induced a significant decrease in apoptotic death and expression of cleaved caspase- 3 and caspase- 9 , indicating suppression of cell death in keloid fibroblasts transfected with the miR-181a mimic, compared with scramble control cells. Conversely, the miR-181a inhibitor enhanced apoptosis and expression of cleaved caspase- 3 and caspase- 9 in keloid fibroblasts. TUNEL staining data showed that miR-181a inhibits human keloid fibroblast apoptosis, consistent with flow cytometry findings (Fig. 3C). Clearly, overexpression of miR-181a inhibits cell apoptosis, while its suppression promotes apoptosis in human keloid fibroblasts.

miR-181 a suppresses PHLPP2 and negatively regulates the AKT pathway in human keloid fibroblasts

Further studies are required to explore the molecular mechanisms linking miR-181a with keloids. PHLPP2 was identified as a potential target of miR-181a from publicly available KARGER 
A

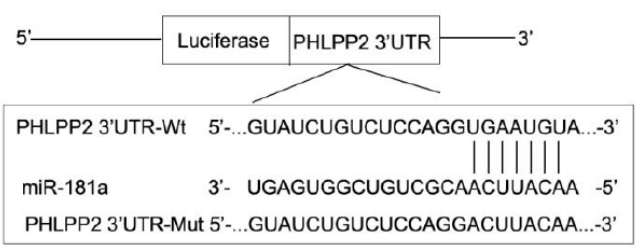

C

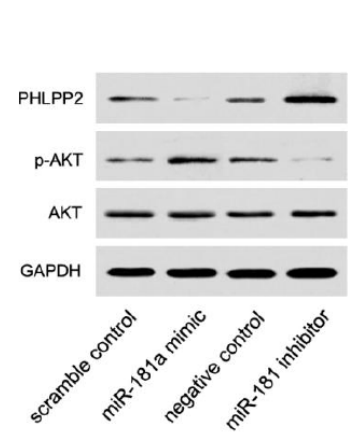

D

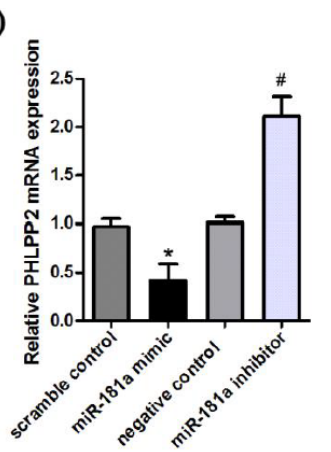

$\mathrm{F}$

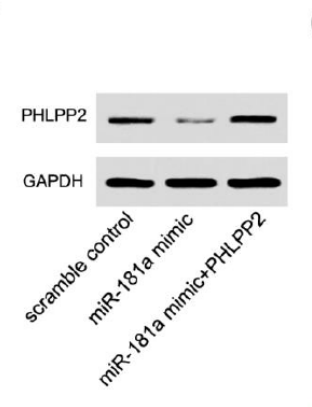

G

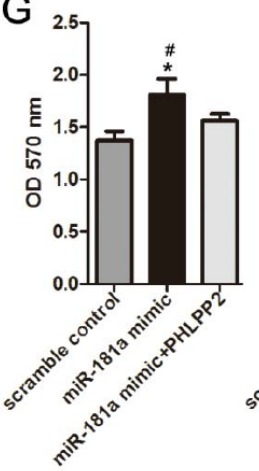

B

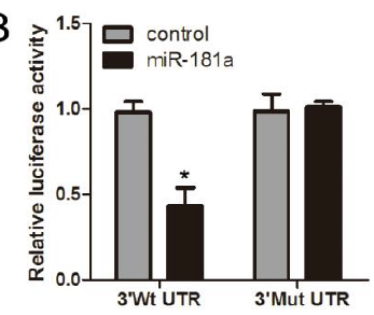

$\mathrm{E}$

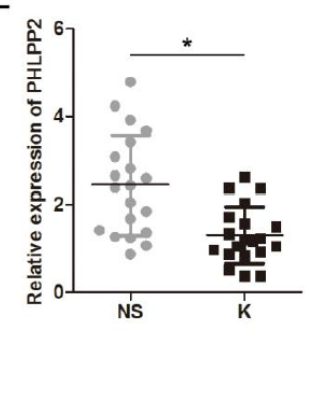

$\mathrm{H}$
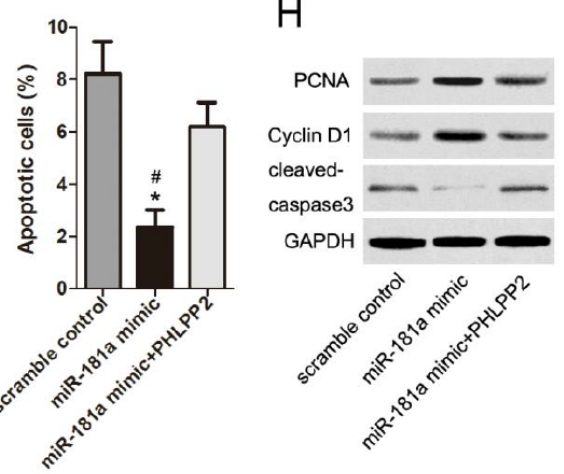

Fig. 4. miR-181a suppresses PHLPP2 and negatively regulates the AKT pathway in human keloid fibroblasts. (A) Schematic representation of the predicted binding sites of miR-181a and PHLPP2. (B) Luciferase activity in keloid fibroblasts cotransfected with miR-181a and PHLPP2-3'UTR Wt or PHLPP2-3'UTR Mut reporter constructs in the presence of miR-181a, relative to control. ${ }^{*} P<0.05$ vs. control group. (C) Western blot analysis of expression of PHLPP2, phosphorylated AKT, AKT, and $\beta$-actin proteins of keloid fibroblasts transfected with miR-181a mimic, miR-181a inhibitor and the respective controls. (D) Expression of PHLPP2 at mRNA level in keloid fibroblasts transfected with miR-181a mimic, miR-181a inhibitor and the respective controls. ${ }^{*} P<0.05$ vs. scramble control group; ${ }^{\#} P<0.05$ vs. negative control group. (E) PHLPP2 expression in normal skin (NS) and keloid tissues (K). (F) Western blot analysis of PHLPP2 expression in different groups of transfected keloid fibroblasts. (G) PHLPP2 counteracts the effects of keloid fibroblasts induced by miR-181a, compared with that in control cells. ${ }^{*} P<0.05$ vs. scramble control group; ${ }^{\#}<0.05$ vs. miR-181a +PHLPP2 group. (H) Western blot analysis of PCNA, cyclin D1 and cleaved caspase 9 expression in different groups of transfected keloid fibroblasts.

databases (TargetScan and miRanda). To confirm whether PHLPP2 is a miR-181a target, a luciferase reporter vector containing the wild-type miR-181a target sequences of PHLPP2 $33^{\prime}$ UTR ( $3^{\prime}$ UTR-Wt) or mutant miR-181a target sequences ( $3^{\prime}$ UTR-Mut) was constructed and luciferase reporter assays performed (Fig. 4A). Constructs were co-transfected with a miR181a mimic or control miRNA into keloid fibroblasts. Luciferase activity of the $3^{\prime} \mathrm{UTR}-\mathrm{Wt}$ reporter gene was significantly decreased after co-transfection with the miR-181a mimic, whereas activity of the mutant reporter gene was not affected, clearly indicating that miR181a binds the PHLPP2 3 'UTR region (Fig. 4B). Subsequent experiments disclosed significant 


\section{Cellular Physiology Cell Physiol Biochem 2016;40:796-806

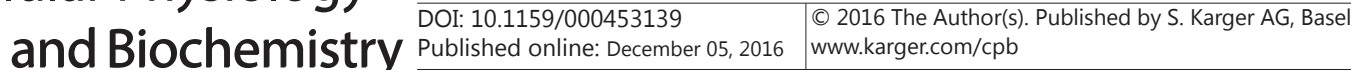 \\ Rang et al.: MiR-181a Regulates Keloid Fibroblast Proliferation and Apoptosis}

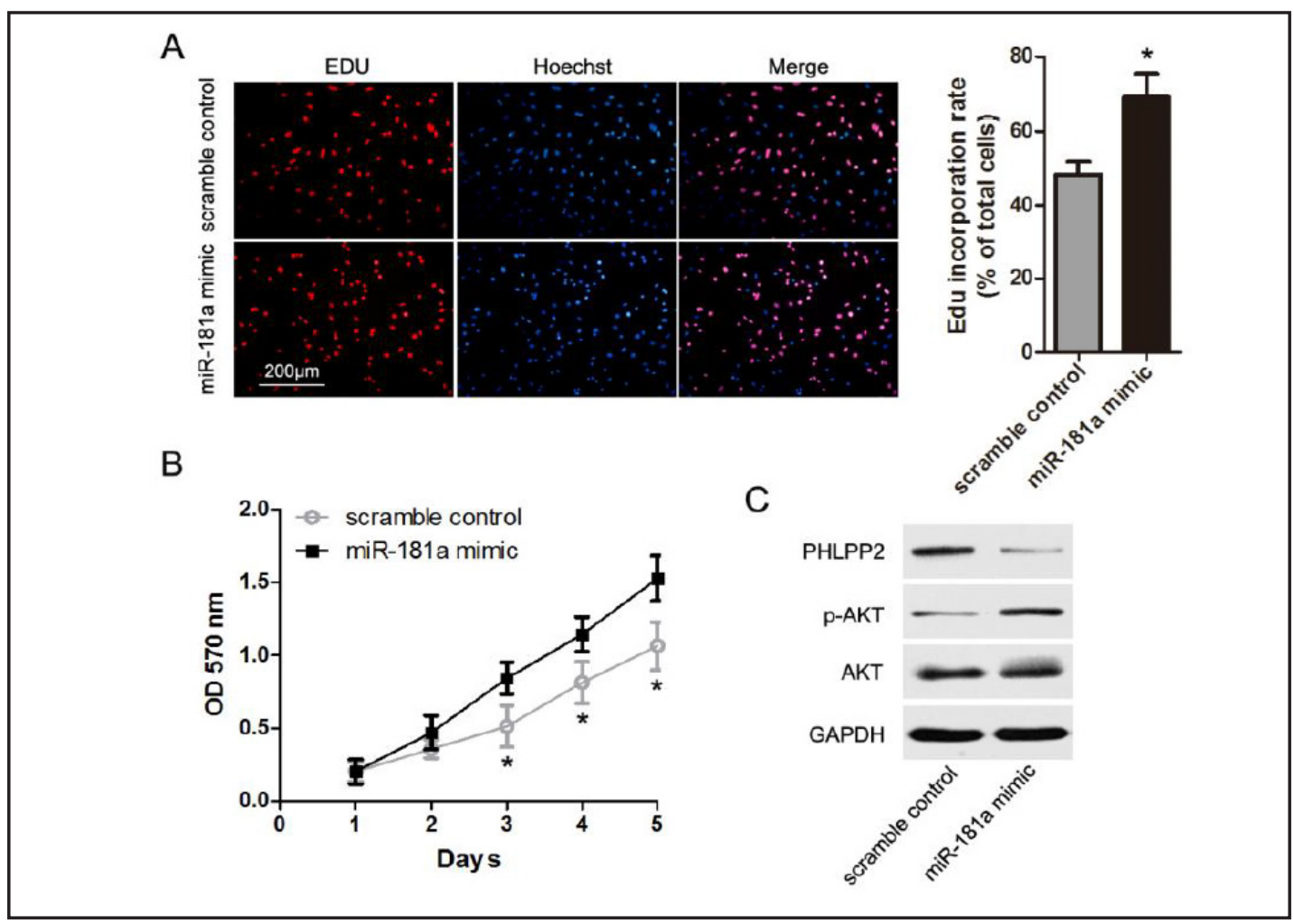

Fig. 5. miR-181a promotes normal skin fibroblast proliferation. (A) EdU incorporation assay. The EdU incorporation rate was expressed as the ratio of EdU-positive cells to total Hoechst 33342-positive cells. (B) Cell proliferation abilities of normal fibroblasts after transfection with miR-181a mimic determined using the CCK-8 assay. (C) Western blot analysis of expression of PHLPP2, phosphorylated AKT and AKT proteins of normal fibroblasts transfected with miR-181a mimic and negative control. ${ }^{*} P<0.05$ vs. scramble control group.

downregulation of PHLPP2 protein and mRNA expression by the miR-181a mimic, and conversely, upregulation by the miR-181a inhibitor, compared to the respective control groups. PHLPP2, a recently characterized member of the PHLPP family and suppressor of AKT signaling, is reported to inhibit cell cycle progression and promote cellular apoptosis [15]. Ectopic miR-181a expression in keloid fibroblasts increased the phosphorylated AKT protein level, whereas depletion of miR-181a decreased phosphorylated AKT protein expression to a significant extent (Fig. 4C and D). We additionally measured PHLPP2 expression in both keloid and normal skin tissues. PHLPP2 expression was suppressed in keloid, compared with normal skin tissues (Fig. 4E). These findings collectively indicate that the expression patterns of miR-181a and PHLPP2 are inversely correlated, supporting the theory that PHLPP2 is a direct downstream target of miR-181a. Given that miR-181a enhances cell proliferation, inhibits apoptosis and suppresses PHLPP2 in keloid fibroblast cells, we speculated that miR181a affects the phenotype of keloid fibroblasts via specific regulation of PHLPP2 $3^{\prime}$ UTR. To examine this hypothesis, we overexpressed PHLPP2 depleted of the $3^{\prime} U T R$ region in keloid fibroblasts and measured the protein levels (Fig. 4F). In addition, we observed its ability of cell proliferation and apoptosis. Notably, increased proliferation and decreased apoptosis were attenuated upon transfection of the PHLPP2 overexpression vector devoid of the $3^{\prime} U T R$ region (Fig. 4G). Accordingly, the expression of PCNA and cyclin D1 was attenuated, while cleaved caspase 9 expression was enhanced in PHLPP2+miR-181a mimic group compared with miR-181a group (Fig. 4H). Based on the findings, we propose that proliferation and apoptosis of keloid fibroblasts are impaired via the miR-181a/ PHLPP2 signaling axis. 


\section{Cellular Physiology Cell Physiol Biochem 2016;40:796-806

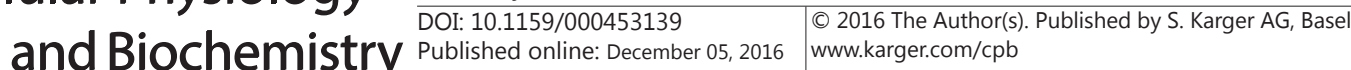 \\ Rang et al.: MiR-181a Regulates Keloid Fibroblast Proliferation and Apoptosis}

miR-181a promotes proliferation of normal skin fibroblasts

We further examined whether overexpression of miR-181a affects the cell phenotype of normal fibroblasts. The EdU incorporation assay was performed to determine the effects of miR-181a on normal fibroblast proliferation. Notably, the number of EdU-positive normal fibroblasts in the miR-181a mimic transfection group was enhanced, relative to that of cells in the scramble control group (Fig. 5A). We further confirmed the effects of miR-181a on cell proliferation using CCK-8 assay (Fig. 5B). In addition, ectopic miR-181a expression in normal fibroblasts made a decrease in PHLPP2 expression and an increase in the phosphorylated AKT at protein levels (Fig. 5C). All data signified that miR-181a acts as a stimulus for abnormal growth.

\section{Discussion}

In this study, we have demonstrated a role of miR-181a in human keloid fibroblast development via promotion of cell proliferation and inhibition of apoptosis. As expected, miR-181a inhibition markedly abrogated the proliferative ability of human keloid fibroblasts and induced apoptosis. At the molecular level, AKT pathways contribute to the miR-181amediated malignant phenotype of human keloid fibroblasts, possibly via inhibition of PHLPP2 expression. The above data clearly support a potential role of miR-181a in the development and progression of human keloid fibroblasts.

Recent evidence has suggested that miRNAs play important roles in fibrosis and they may be useful targets for treatment of the disease [16]. miR-181a is a multifaceted miRNA implicated in many cellular processes, including cell fate determination, proliferation and invasion. Earlier, Shi et al. [17] showed that miR-181a functions as a tumor suppressor by triggering growth inhibition, inducing apoptosis, and suppressing invasion in glioma cells. In contrast, miR-181a is reported to promote G1/S transition and cell proliferation in pediatric acute myeloid leukemia by targeting ATM [18]. Jianwei and colleges demonstrated that miR-181a improves proliferation and invasion and suppresses apoptosis of osteosarcoma cells [19]. Data from the current study showed that miR-181a is increased in human keloid fibroblasts, although its functions and molecular mechanisms remain to be established. Our findings provide comprehensive evidence of the functional, mechanical and clinical significance of this molecule. Firstly, we confirmed increased expression of miR-181a in human keloid tissues and fibroblasts, compared to normal skin tissues and fibroblasts, respectively. Next, we showed that miR-181a overexpression promotes cell proliferation and inhibits apoptosis, whereas miR-181a inhibition has the opposite effect. Further research is required to elucidate the molecular pathways mediating the different biological effects and targets of miR-181a in human keloid fibroblasts.

PHLPP2, a phosphatase essential for specific and effective termination of oncogenic AKT signaling, was identified as a target gene of miR-181a in the current study [20,21]. PHLPP2, a tumor suppressor that inhibits cell growth and induces apoptosis in cancer cell lines [15], is located at 16q22.3, a region that undergoes loss of heterozygosity (LOH) in breast and ovarian cancers, prostate cancer, and hepatocellular carcinoma [22]. However, the expression patterns and biological functions of PHLPP2 in human keloid fibroblasts remain to be established. PHLPP2 was determined as a target of miR-181a using bioinformatics analysis software, which was further verified using a double-luciferase reporter assay. Moreover, PHLPP2 was downregulated in human keloid tissues, compared with normal skin tissues. PHLPP2, a characterized member of the PHLPP family and suppressor of AKT signaling, inhibits cell cycle progression and induces apoptosis in various cancer cell types [21]. Accordingly, we examined the expression of phosphorylated and total AKT in keloid fibroblasts transfected with a miR-181a mimic, miR-181a inhibitor or the irrespective controls to clarify the pathway associated with PHLPP2 activity in keloid fibroblasts. The miR181a mimic suppressed PHLPP2 expression and enhanced phosphorylated AKT expression, 


\section{Cellular Physiology Cell Physiol Biochem 2016;40:796-806 \begin{tabular}{l|l|l|} 
and BOI: 10.1159/000453139 & $\begin{array}{l}\text { C) 2016 The Author(s). Published by S. Karger AG, Basel } \\
\text { www.karger.com/cpb }\end{array}$
\end{tabular} \\ Rang et al.: MiR-181a Regulates Keloid Fibroblast Proliferation and Apoptosis}

suggesting that the miRNA inhibits apoptosis via blocking the phosphatidylinositol 3-kinase/ AKT pathway. The miR-181a inhibitor exerted the opposite effects. Moreover, the miR-181a mimic promoted normal skin fibroblast cell proliferation. Our collective data suggest that miR-181a negatively regulates PHLPP2 expression, and increases cell proliferation and inhibits apoptosis through activating the AKT pathway in keloid fibroblasts.

In summary, miR-181a promotes cell proliferation and inhibits apoptosis through suppressing PHLPP2 expression, leading to activation of the AKT pathway, and consequently, accelerated cell growth of keloid fibroblasts. Our findings support the potential utility of both miR-181a and PHLPP2 as novel biomarkers and targets for diagnostic and therapeutic approaches for keloids.

\section{Acknowledgements}

This work was supported by the National Natural Science Foundation of China (81101231).

\section{Disclosure Statement}

The authors declare there are no conflicts of interest.

\section{References}

1 Kashiyama K, Mitsutake N, Matsuse M, Ogi T, Saenko VA, Ujifuku K, Utani A, Hirano A, Yamashita S: Mir196a downregulation increases the expression of type i and iii collagens in keloid fibroblasts. J Invest Dermatol 2012;132:1597-1604.

2 Han JY, Han JF, Yu DM, Xiao JL, Shang Y, Hao LJ: Association of ADAM33 gene polymorphisms with keloid scars in a northeastern Chinese population. Cell Physiol Biochem 2014;34:981-987.

3 Jumper N, Hodgkinson T, Arscott G, Har-Shai Y, Paus R, Bayat A: The aldo-keto reductase akr1b10 is upregulated in keloid epidermis, implicating retinoic acid pathway dysregulation in the pathogenesis of keloid disease. J Invest Dermatol 2016;136:1500-1512.

4 Bartel DP: Micrornas: genomics, biogenesis, mechanism, and function. Cell 2004;116:281-297.

5 Wienholds E, Plasterk RH: Microrna function in animal development. Febs Lett 2005;579:5911-5922.

6 Hwang HW, Mendell JT: Micrornas in cell proliferation, cell death, and tumorigenesis. Br J Cancer 2006;94:776-780.

7 Zhang GY, Wu LC, Liao T, Chen GC, Chen YH, Zhao YX, Chen SY, Wang AY, Lin K, Lin DM, Yang JQ Gao WY, Li QF: A novel regulatory function for mir-29a in keloid fibrogenesis. Clin Exp Dermatol 2016;41:341-345.

8 Cheng J, Yu H, Deng S, Shen G: Microrna profiling in mid- and late-gestational fetal skin: implication for scarless wound healing. Tohoku J Exp Med 2010;221:203-209.

9 Liu Y, Yang D, Xiao Z, Zhang M: Mirna expression profiles in keloid tissue and corresponding normal skin tissue. Aesthetic Plast Surg 2012;36:193-201.

10 Dong X, Zhang C, Ma S, Wen H: Mast cell chymase in keloid induces profibrotic response via transforming growth factor-beta1/smad activation in keloid fibroblasts. Int J Clin Exp Pathol 2014;7:3596-3607.

11 Li P, He QY, Luo CQ: Overexpression of mir-200b inhibits the cell proliferation and promotes apoptosis of human hypertrophic scar fibroblasts in vitro. J Dermatol 2014;41:903-911.

12 Chang RM, Yang H, Fang F, Xu JF, Yang LY: Microrna-331-3p promotes proliferation and metastasis of hepatocellular carcinoma by targeting ph domain and leucine-rich repeat protein phosphatase. Hepatology 2014;60:1251-1263.

13 Wang F, Zhao XQ, Liu JN, Wang ZH, Wang XL, Hou XY, Liu R, Gao F, Zhang MX, Zhang Y, Bu PL: Antagonist of microrna-21 improves balloon injury-induced rat iliac artery remodeling by regulating proliferation and apoptosis of adventitial fibroblasts and myofibroblasts. J Cell Biochem 2012;113:2989-3001. 


\section{Cellular Physiology Cell Physiol Biochem 2016;40:796-806 \begin{tabular}{l|l|l} 
DOI: 10.1159/000453139 & $\begin{array}{l}\text { O 2016 The Author(s). Published by S. Karger AG, Basel } \\
\text { www.karger.com/cpb }\end{array}$
\end{tabular} \\ Rang et al.: MiR-181a Regulates Keloid Fibroblast Proliferation and Apoptosis}

14 Liu Y, Wang X, Yang D, Xiao Z, Chen X: Microrna-21 affects proliferation and apoptosis by regulating expression of pten in human keloid fibroblasts. Plast Reconstr Surg 2014;134:561e-573e.

15 Brognard J, Sierecki E, Gao T, Newton AC: Phlpp and a second isoform, phlpp2, differentially attenuate the amplitude of akt signaling by regulating distinct akt isoforms. Mol Cell 2007;25:917-931.

16 Steven O'Reilly: MicroRNAs in fibrosis: opportunities and challenges. Arthritis Res Ther 2016;18:11.

17 Shi L, Cheng Z, Zhang J, Li R, Zhao P, Fu Z, You Y: Hsa-mir-181a and hsa-mir-181b function as tumor suppressors in human glioma cells. Brain Res 2008;1236:185-193.

18 Liu X, Liao W, Peng H, Luo X, Luo Z, Jiang H, Xu L: Mir-181a promotes g1/s transition and cell proliferation in pediatric acute myeloid leukemia by targeting atm. J Cancer Res Clin Oncol 2016;142:77-87.

19 Jianwei Z, Fan L, Xiancheng L, Enzhong B, Shuai L, Can L: Microrna 181a improves proliferation and invasion, suppresses apoptosis of osteosarcoma cell. Tumour Biol 2013;34:3331-3337.

20 Nowak DG, Cho H, Herzka T, Watrud K, DeMarco DV, Wang VM, Senturk S, Fellmann C, Ding D, Beinortas T, Kleinman D, Chen M, Sordella R, Wilkinson JE, Castillo-Martin M, Cordon-Cardo C, Robinson BD, Trotman LC: Myc drives pten/trp53-deficient proliferation and metastasis due to il6 secretion and akt suppression via phlpp2. Cancer Discov 2015;5:636-651.

21 Wei XE, Zhang FY, Wang K, Zhang QX, Rong LQ: Assembly of the fkbp51-phlpp2-akt signaling complex in cerebral ischemia/reperfusion injury in rats. Brain Res 2014;1566:60-68.

22 Brognard J, Newton AC: Phlipping the switch on akt and protein kinase c signaling. Trends Endocrinol Metab 2008;19:223-230. 